\title{
IMAGE EDGE DETECTION USING YUV COLOR SPACE: AN ALTERNATIVE TO RGB COLOR SPACE
}

\author{
Aliyu Lawan Musa', Sani Ahmad Muhammad², Abubakar Sadiq Muhammad ${ }^{3}$, Abubakar \\ Abdullahi Umar ${ }^{4}$, Khadija Mahmoud Sani ${ }^{5}$ and Sulaiman Inuwa Tofa ${ }^{6}$ \\ ${ }^{1,3,4,6}$ Department of Computer Engineering, and ${ }^{2,5}$ Department of Computer Science, School of \\ Technology, Kano State Polytechnic, Nigeria \\ E-mail: Aliyulmusa@kanopoly.edu.ng,Abbankhairat@gmail.com
}

\begin{abstract}
The concept of edge detection is an aspect of image processing that attracts lot of attention as it is known to significantly reduce the amount of data and filters out useless information in an image. Boundaries of an image are usually characterized by edges, which in turn preserve the important structural properties in an image. Color is a property that is imminent and which simplifies description, object identification and extraction from scene thereby making colour image edge detection to have widespread in colour image segmentation. This paper reviews the method of $R G B$ image edge detection with an improvement on colour image edge detection by transformation from RGB colour space to $Y U V$ colour space, processing the $Y$ component of the YUV image accompanied with histogram equalization transform of the processed image. The experiment results show that the improved method provides a faster method for colour image detection whilst retaining more features when compared to RGB image detection.
\end{abstract}

Keyword: Histogram equalization, colour image, YUV colour space and edge detection. 


\section{INTRODUCTION}

Edge detection usually provides sharp transitions of intensity of colour within an image. These transitions are characteristic of object edges. Once edges of an object are detected other processing such as region segmentation, text finding, and object recognition becomes easier and possible. A number of researchers in the past few decades have concentrated on devising algorithms for grayscale image understanding (Jain, 1989). With the advent of powerful personal computers, it is now possible and practical to move to the more computationally intensive realm of color image understanding. There are many benefits in using color images. Task such as object location, processing, and the possibility of processing images that are more complex is viable from the increase in the quantity of information resulting from the colored image (Jain, 1989). Statistics have shown that about $90 \%$ of edge information in coloured images is the same in gray images but it has to be pointed out that no edges can be detected in gray values images when neighboring objects have equal intensities but different hues. Therefore, an object that cannot be distinguished in gray value images are treated as one big object in the scene thereby becoming an obstacle to the task of object identification in the scene. This also implies that $10 \%$ of the edge information in the colour has not been detected, thus making it essential to research the problem of colour image detection (J Fan, 2001) (S K Naik, 2006).

A lot of researchers have put forward much arithmetic on RGB image edge detection whose basic steps include expanding the edge detection to the three components of RGB colour space and combining the edge of the three components by definite logic algorithm and obtaining the colour image edge (Zhongshui QU, 2010). The common shortcomings of these RGB image detection approach is the low speed and colour losses after each component is processed ( $\mathrm{R} \mathrm{C}$ Gonzalez, 2003). A colour space known to have closeness to the colour vision characteristics than other colour spaces are the YUV colour space (R D Dony, 1999). It is a color space in which each of the components exist independently, the luminance component Y provides a principal advantage in image processing such that an image in the YUV space has only the $\mathrm{Y}$ component decoupled for processing by a number of algorithms whilst retaining its color components $(\mathrm{U}, \mathrm{V})$ thus making it adopted for colour image detection.

This paper proposes a method for image detection in the YUV space and improves it according to histogram equalization using three edge detection algorithms. The Gradient operator (horizontal and vertical), Laplacian operator and Laplacian with control parameter $(\alpha)$. The overall framework of the proposed approach based on YUV colour image detection method is organized as follows: Section1 presents the color space and models, Section 2 presents the operators employed for the colour image detection and RGB edge detection process, Section 3 presents YUV image edge detection method and Section 4 presents Histogram equalization method. Experimental results and discussion are given in sections 5 and 6.

\section{I- RGB AND YUV COLOR SPACE}

Color may be defined by its attributes of brightness, hue and purity for humans. A color space is a method by which we can specify, create and visualize color. A color is usually specified using three coordinates that are parameters describing the position of the color within the color space being used. 


\section{a- RGB Color Space}

RGB is an additive colour model in which the primaries $\operatorname{Red}(\mathrm{R})$, Green $(\mathrm{G})$, Blue(B) lights are added together to produce a wide array of colours. The RGB colour model has a solid theory behind it based on human perception of colour and light indicating that its characteristics fail to match the intuition of human psychology, which brings along with it the aspect of lightness of the colour and the amount of colour used to colour a specific region. Also, the distance between any two colour points is not equal with the difference of vision characteristics, thereby resulting in drawbacks in obtaining the three characteristics of colour (hue, luminance and saturation) through RGB data.

\section{b- YUV Colour Space}

The YUV color model is the basic color model used in analogue colour TV broadcasting. It served as the recoding of RGB for transmission efficiency by minimizing bandwidth and for downward compatibility with black and white television. YUV model defines a colour space comprising of nonlinear luma/chroma in which one component (Y) shows the monochrome portion of the image that determines image lightness and the two other components show the colour in the image. Owing to the fact that human visual system is far more sensitive to difference in lightness than in colour and the non-linear eye's perception of a gamma correction on the luminance (brightness) to form luma (Y) allows obtaining the attributes of colour images since each component is independent of the other.

Conversion between RGB and YUV Color Space

$$
\begin{aligned}
& U=0.493(B-Y) \\
& V=0.877(R-Y)
\end{aligned}
$$

So the conversion between RGB and YUV color space is given by the equations:

$$
\begin{aligned}
& \left(\begin{array}{l}
Y \\
U \\
V
\end{array}\right)=\left(\begin{array}{ccc}
0.299 & 0.587 & 0.114 \\
-0.147 & -0.289 & 0.436 \\
0.615 & -0.515 & 0.100
\end{array}\right)\left(\begin{array}{l}
R \\
G \\
B
\end{array}\right) \\
& \left(\begin{array}{l}
R \\
G \\
B
\end{array}\right)=\left(\begin{array}{ccc}
1.000 & 0.000 & 0.140 \\
1.000 & -0.369 & 0.581 \\
1.000 & 2.029 & 0.000
\end{array}\right)\left(\begin{array}{l}
Y \\
U \\
V
\end{array}\right)
\end{aligned}
$$

\section{II- ANALYSIS OF RGB COLOR IMAGE EDGE DETECTION METHODS}

\section{a. Component Gradient Operator}

The function of component gradient operator popularly referred to as Sobel Operator is to process the decomposed components of the RGB colour image (R. Subban, 2013) (S K Naik, 2006). The kernels of the component gradient operator method are designed to respond maximally to edges running vertically and horizontally relative to the pixel grid, one kernel for each of the two perpendicular orientation is employed to provide the grayscale version 
of each R, G, and B component given by an RGB colour image.

The operation of the gradient operator of colour image edge detection method in RGB color space consists of three steps:

I. The $\mathrm{R}, \mathrm{G}$ and $\mathrm{B}$ component is given by an RGB color image.

II. The kernel of the gradient operator will be applied to obtain separate measurements of the gradient components in each orientation $\left(\mathbf{G}_{\mathbf{x}}\right.$ and $\left.\mathrm{G}_{\mathbf{y}}\right)$

III. The result image is given by the processed $\mathrm{R}, \mathrm{G}$ and $\mathrm{B}$ component.

Suppose $\mathrm{p}$ is an arbitrary vector in RGB color space:

$$
P=\left(\begin{array}{l}
P_{X} \\
P_{Y} \\
P_{Z}
\end{array}\right)=\left(\begin{array}{l}
R \\
G \\
B
\end{array}\right)
$$

This equation indicates that the components of $\mathrm{P}$ are simply the RGB components of a color image at a point. Taking into account the fact that the colour components are a function of coordinates $(\mathrm{x}, \mathrm{y})$ thus equation(5) can be rewritten as using the notion:

$$
P=\left(\begin{array}{l}
P_{R}(x, y) \\
P_{G(x, y)} \\
P_{B(x, y)}
\end{array}\right)=\left(\begin{array}{l}
R_{(x, y)} \\
G_{(x, y)} \\
B_{(x, y)}
\end{array}\right)
$$

The Sobel operation will be carried out by the 2-D convolution kernel whose mask is as shown in figure 1(a) with equation given for $\left(\mathbf{G}_{\mathbf{x}}\right)$ in equation (7)

$G_{x}^{P}=(P(x-1, y-1)+2 P(x-1, y)+P(x-1, y-1))-$

$(P(x+1, y-1)+2 P(x+1, y)+P(x+1, y+1))$

\begin{tabular}{|l|l|l|}
\hline-1 & 0 & +1 \\
\hline-2 & 0 & +2 \\
\hline-1 & 0 & +1 \\
\hline
\end{tabular}

\begin{tabular}{|l|l|l|}
\hline+1 & +2 & +1 \\
\hline 0 & 0 & 0 \\
\hline-1 & -2 & -1 \\
\hline
\end{tabular}

Figure 1(a) Mask used by Sobel operator

\section{b. Laplacian operator}

The Laplacian (second derivative) is an isotropic filter, which is rotation invariant. The output of filtering then rotating is same as the output of rotating then filtering. Its principal objective is to highlight transition intensities. The operation will be carried out by the 2-D convolution kernel whose mask is as shown in figure 1(b) with equation given for $(\mathbf{x}, \mathbf{y})$ components in equation (8). 


$$
\begin{aligned}
& \Delta^{2} f=\frac{\partial^{2} f}{\partial x^{2}}+\frac{\partial^{2} f}{\partial y^{2}} \\
& \Delta^{2} f(x, y)=f(x+1, y)+f(x-1, y)+f(x, y+1)+f(x, y-1)-4 f(x, y)
\end{aligned}
$$

\begin{tabular}{|c|c|c|}
\hline 0 & 1 & 0 \\
\hline 1 & -4 & 1 \\
\hline 0 & 1 & 0 \\
\hline
\end{tabular}

\begin{tabular}{|c|c|c|}
\hline 1 & 1 & 1 \\
\hline 1 & -8 & 1 \\
\hline 1 & 1 & 1 \\
\hline
\end{tabular}

Figure 1(b) Mask used by Laplacian operator

\section{c. Laplacian with controlled parameter}

This is a predefined 2D filter created with a controlled parameter $(\alpha=0.5)$, the parameter is responsible for controlling the shape of the Laplacian. The kernel is as shown below:

$$
\Delta^{2}=\frac{4}{\alpha+1}\left[\begin{array}{ccc}
\frac{\alpha}{4} & \frac{1-\alpha}{4} & \frac{\alpha}{4} \\
\frac{1-\alpha}{4} & -1 & \frac{1-\alpha}{4} \\
\frac{\alpha}{4} & \frac{1-\alpha}{4} & \frac{\alpha}{4}
\end{array}\right]
$$

\section{II- YUV COLOUR IMAGE DETECTION}

The basic idea of the YUV color image edge detection process is to only process the Y component. Figure 1(c) described the Flow chart representation of proposed approach.

\section{IV- HISTOGRAM EQUALISATION}

Histogram equalization is an aspect of image enhancement that involves direct manipulation of image pixels in the spatial domain for the purpose of highlighting interesting detail in images, removing noise from images and making images more visually appealing.

The histogram of an image shows us the distribution of gray levels in the image. The spreading out of the frequencies in an image (or equalizing the image) is a simple way to improve dark or washed out images. The formula for histogram equalization is given as:

$$
\begin{gathered}
S_{k}=T\left(r_{k}\right) \\
=(L-1) \sum_{j=0}^{k} p_{r}\left(r_{k}\right)
\end{gathered}
$$




$$
=\frac{(L-1)}{M N} \sum_{j=0}^{k}\left(n_{j}\right)
$$

Where

$r_{k}=$ Input intensity with $2^{k}$ intensity level, $S_{k}=$ Processed intensity

$n_{j}=$ number of pixel in the image with intensity value $r_{k}$ and $\mathbf{L}=$ intensity level $\left(2^{k}\right)$

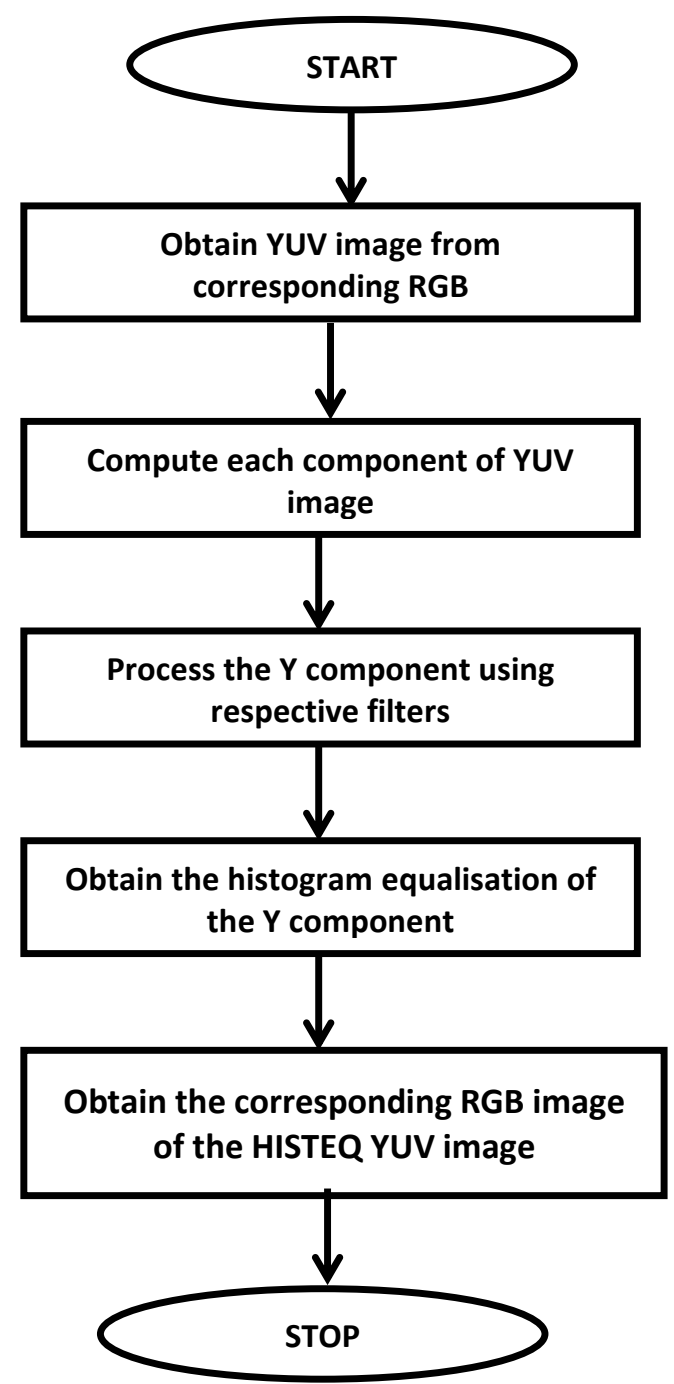

Figure 1(c): Flowchart for YUV image detection

\section{EXPERIMENTAL RESULTS}

The proposed algorithm has been experimented and researched on color images using MATLAB (2018a). In the experiment, one color image (jpg format, $450 \times 600 \times 3$ ) was used as base image as shown in Figure 2. The results of the experiment are shown in Figures 2(a,b), $3(a, b), 4(a, b)$ and figure 5 . 


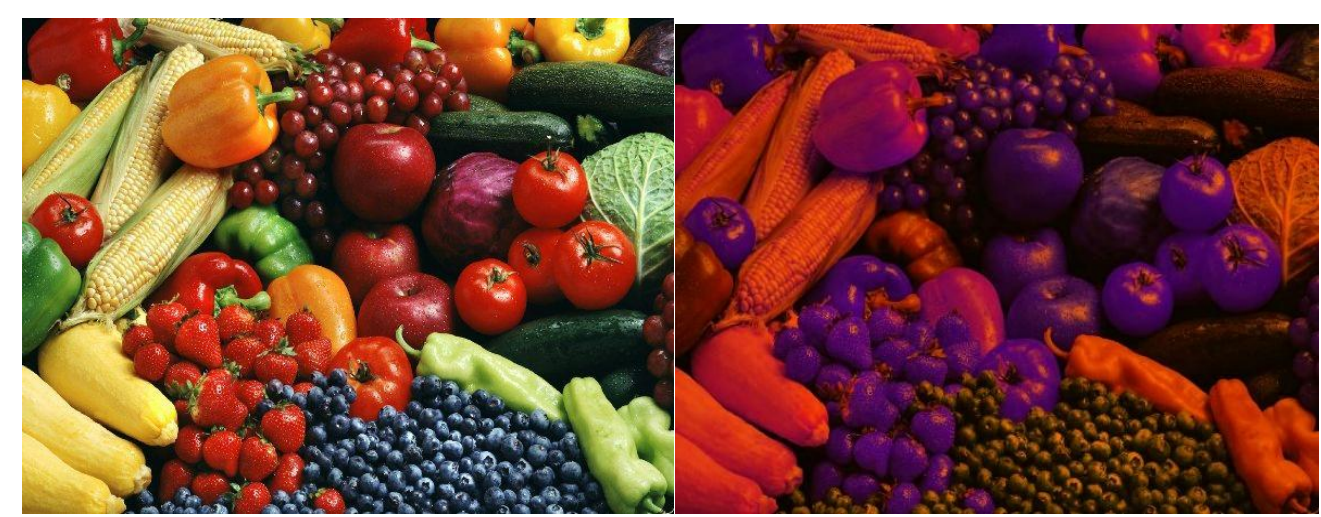

Figure 2(a): Original Colour image and 2(b) Equivalent RGB image in YUV colour space
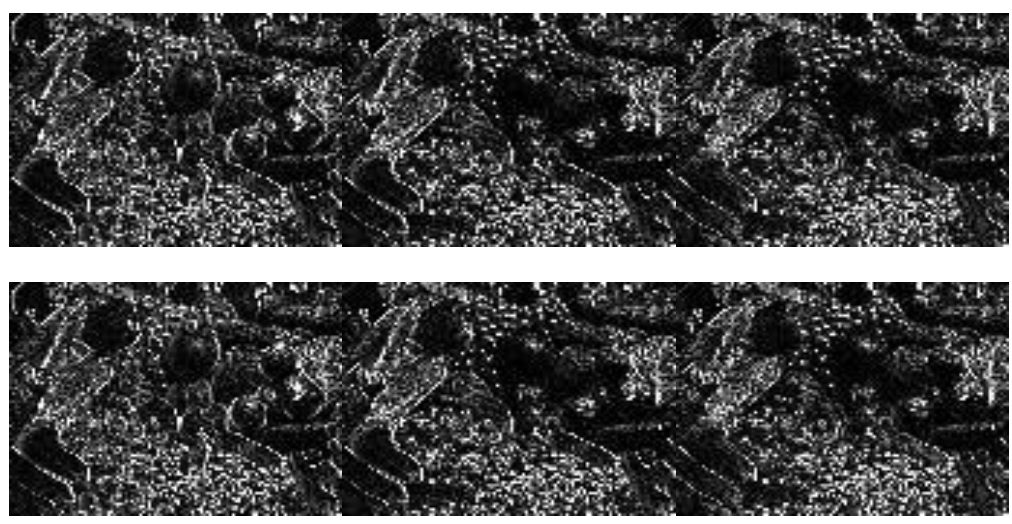

Figure 2(c): Horizontal and vertical Sobel operator on R, G and B components

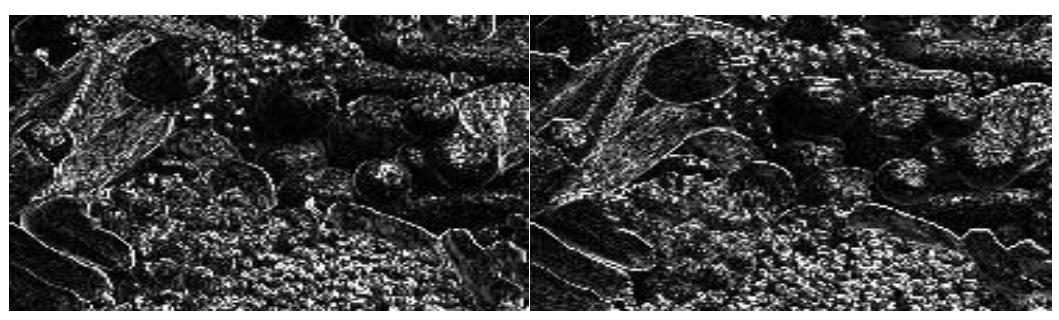

Figure 2(d): Horizontal and vertical Sobel operator on Y component of YUV image

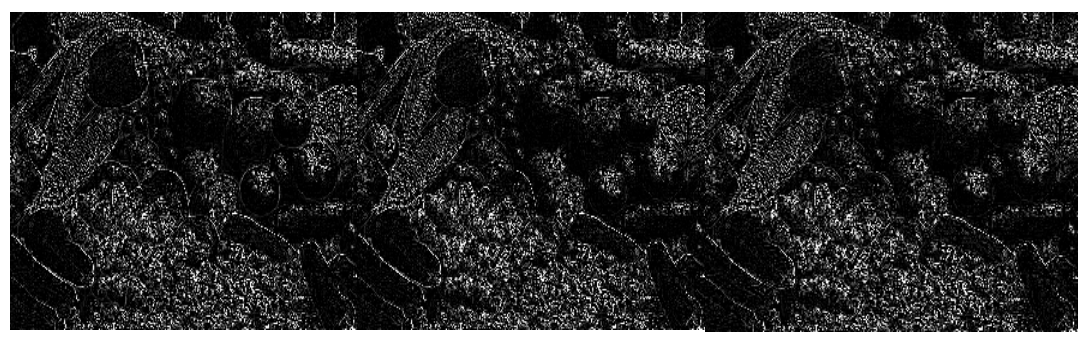




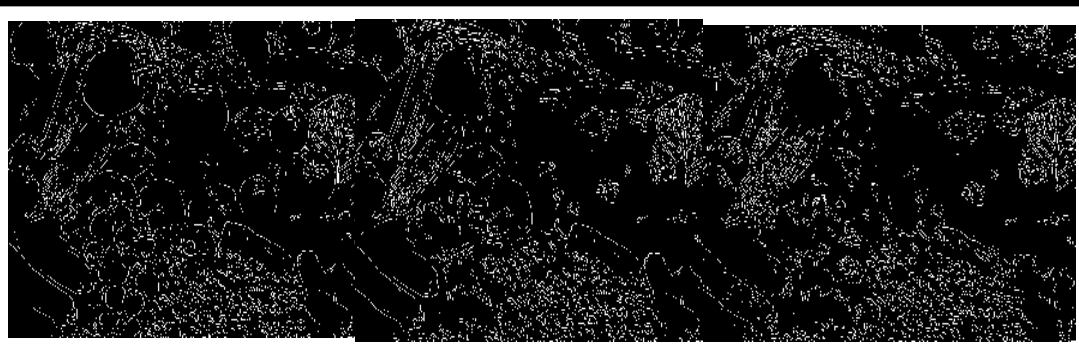

Figure 3(a): Laplacian/Laplacian ( $\alpha$ ) operator on R, G, and B components

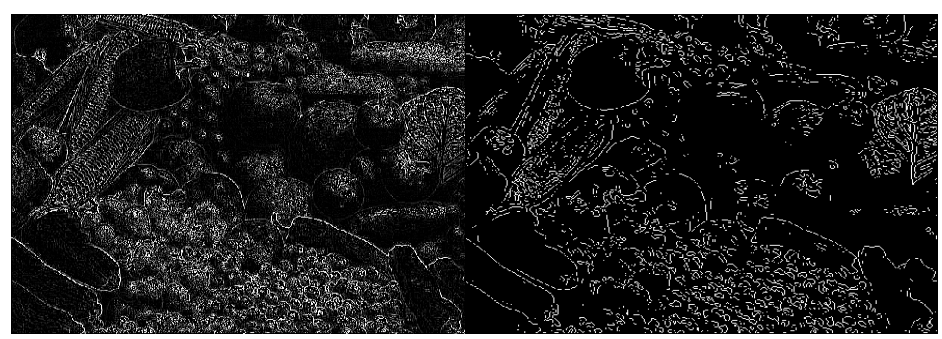

Figure 3(b): Laplacian/Laplacian ( $\alpha$ ) operator on Y component of YUV image

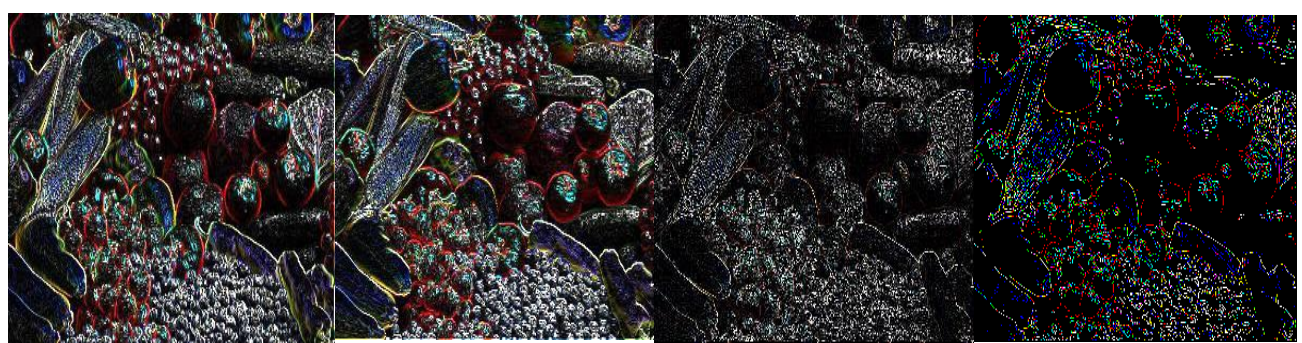

Figure 3(c): Resulting processed RGB image

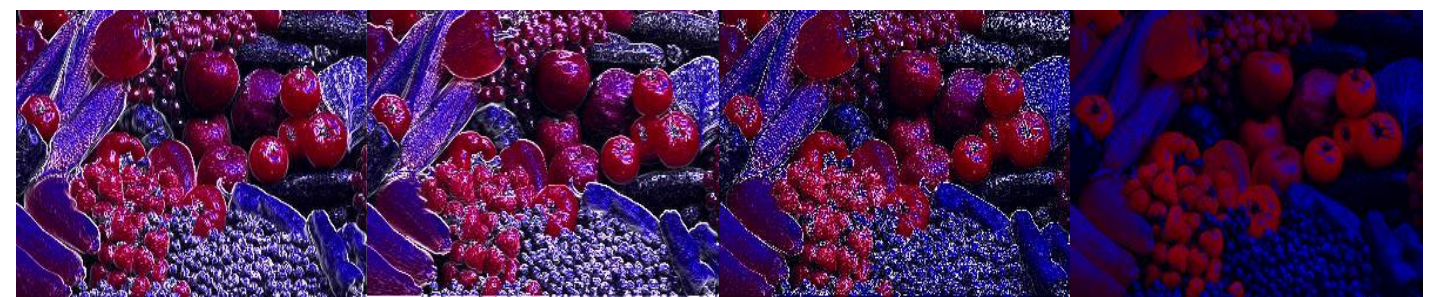

Figure 3(d): Resulting Y component in YUV image

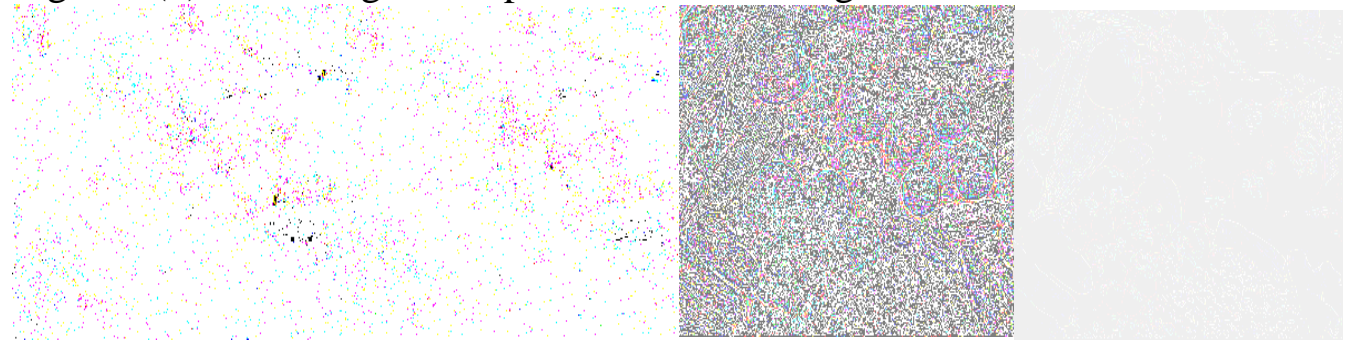

Figure 4(a): Histogram equalized RGB processed image 
International Journal of Advanced Academic Research (Sciences, Technology and Engineering) | ISSN: 2488-9849

Journal DOI: 10.46654/ij.24889849

Vol. 6, Issue 7 (July, 2020) | www.ijaar.org

Article DOI: 10.46654/ij.24889849.e6724
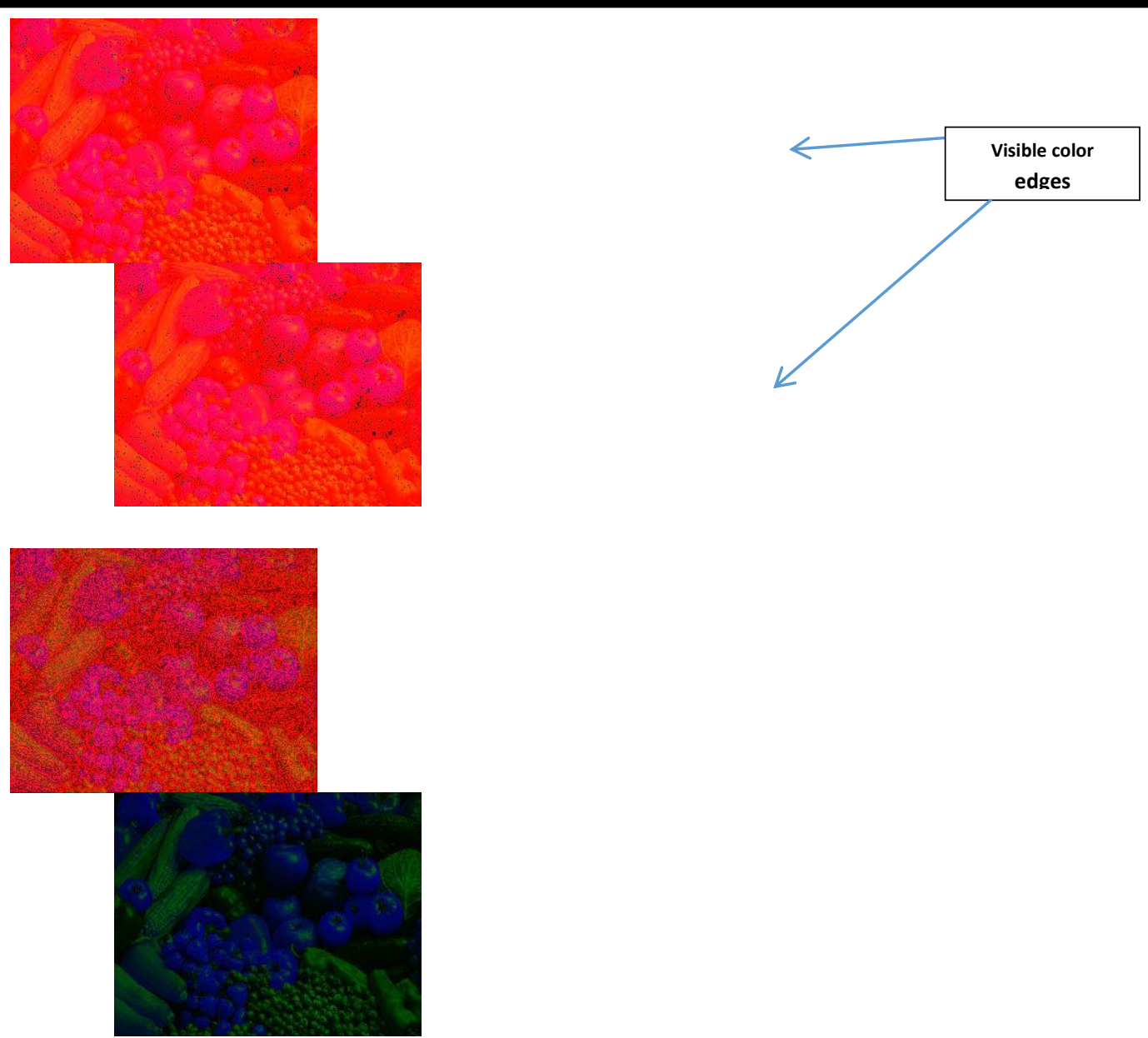

Figure 4(b): Histogram equalized Y component processed YUV image
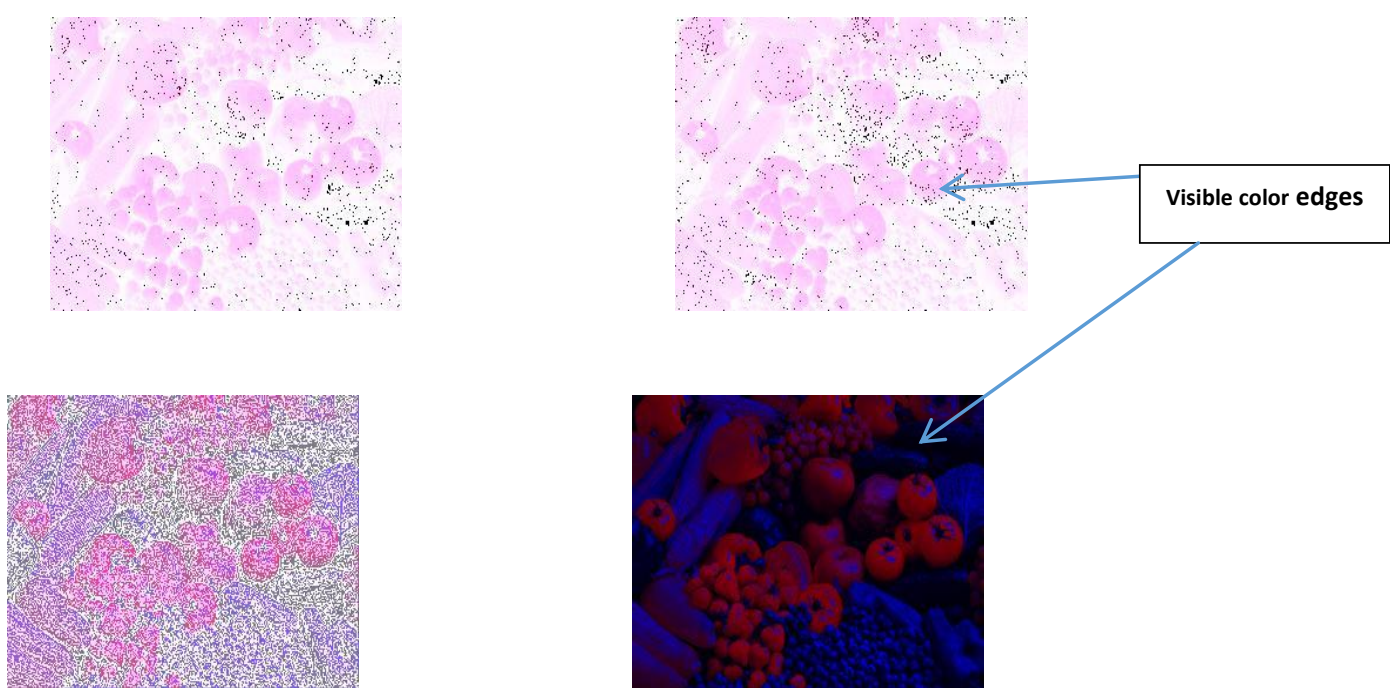

Figure 4(c): Corresponding HISTEQY component processed YUV image in RGB colour space 


\section{VI- DISCUSSION}

Figure 2(a) shows the original image in RGB colour space. Figure 2(b) shows the corresponding RGB in YUV colour space. Figure 2(c) shows the result of horizontal and vertical Sobel operator on individual components of RGB. Figure 2(d) shows the result of horizontal and vertical Sobel operator on Y- component of YUV image. Figure 3(a) shows the result of Laplacian/Laplacian $(\alpha)$ operator on each RGB component. Figure 3(b) shows the result of Laplacian/Laplacian $(\alpha)$ operator on Y-component of YUV image. Figure 3(c) shows the result corresponding processed RGB image using operators. Figure 3(d) shows the result of the processed YUV image. Figure 4(a) shows the result of Histogram equalization on processed RGB images. Figure 4(b) shows the result of Histogram equalization on processed YUV image. Figure 4(c) shows result of the corresponding histogram equalized YUV image in RGB colour space. The experiment employs the use of three operators Sobel (horizontal and vertical), Laplacian and Laplacian with controlled parameter $(\alpha=0.5)$. The Laplacian with controlled parameter is found to reduce noises and stresses more edges in each image model (Sobel and Laplacian) when used for the respective components (R, G, B and Y) as seen in figure 3(a) and 3(b). The results obtained show that operation on the RGB colour space has to be carried out on each component (R, G and B) of the colour image. This appears to be time consuming for each operator especially if large numbers of colour images are to be processed. However, the processed Y-component of the YUV image produces the same results as that of processing the individual components of the RGB image when passed through the same operator making the colour edge detection method simpler and faster. The RGB image is seen to lose most of its

colour features after operated on by the algorithms as in Figure 3(d), Similarly, the histogram equalization of the processed RGB image contains redundant information of the edges in the image if compared to the histogram equalization of the Y-processed YUV image which maintains a large portion of its colour and edges. The resulting RGB image of the YUV histogram equalization is also noticed to maintain the colour edges in it.

\section{CONCLUSION}

The proposed algorithm is found to be faster and simpler in edge detection of colour image by processing of a single component $(\mathrm{Y})$ of the YUV image as compared to the three components of the RGB image. The gray edges are found to be more pronounced using the Laplacian with a controlled element, similarly coloured edges are detected effectively and more exact based on the proposed method. In conclusion, the work has proven the significance of using other color space in color image processing and the possibility of enhancing the visibility of edges using the histogram equalization. 


\section{REFERENCES}

Al-Juboori, A. M. (2005). Color Image Enhancement Using Histogram Modification. Msc Thesis. AL-Gadesiy University.

Funtanil, L. A. (n.d.). GIS pattern Recognition and Rejection analysis Using Matlab. Msc Thesis. Texas.

G V Rakesh, T. S. (2012). YCoCg color Image Edge Detection. International Journal Of Engineering Research and Application, 152-156.

J Fan, W. G. (2001). An Improved Automatic Isotropic Color Edge Detection Technique. Pattern Recognition Letters, 1419-1429.

Jain, A. K. (1989). Fundamentals of Digital Image Processing. Englewood Cliffs NJ: Prentice Hall Inc.

K. B Shaik, P. G. (2015). Comparative study of skin color detection and segmentation in HSV and YCbCr. International conference on Recent Trends in Computing . ICRTC.

M Podpora, G. K. (2014). YUV vs RGB- Choosing a Color Space for Human-Machine Interaction. Federated Conferences on Computer Science and Information Systems (pp. 29-34). ACSIS.

P E Trahanias, A. N. (1993). Color Detection Using Vector OrderStatistics. IEEE Transaction on Image Processing, 259-264.

R C Gonzalez, R. E. (2003). Digital Image Processing. New York: Prentice Hall.

R D Dony, S. W. (1999). Edge Detection on color Images using RGB vector angles. IEEE conference on Electrical and computer Engineering, (pp. 687-692).

R. Subban, R. M. (2013). Combining Color Spaces for Human Skin Detection in Color Images using skin cluster classifier. Intl. Conference on Advances in Recent Technologies in Elecetrical and Electronics. ACEE.

Raman Maini, H. A. (2012). Study and Comparison of Various Image Edge Detection Techniques. International Journal of Image Processing, 3(1).

S K Naik, C. A. (2006). Standadization of Edge magnitude in color Images. IEEE Transactions on Image Processing, 2588-2595.

S, R. M. (2006). Performance Evaluation of Prewitt Edge Detector For Noisy Images. GVIP Journal, 6(3).

S.K, K. (2002). GPS for Geometric Correction of remotely sensed imagery: Possibilities after Terminationof SA. India International Center New Delhi.

Wesolkowski, S. B. (1999). Color Image Edge Detection and Segmentation: A comparison of the Vector angle and the Euclidean Distance color similarity Measures. Msc Thesis. Canada: University of Waterloo. 
International Journal of Advanced Academic Research (Sciences, Technology and Engineering) | ISSN: 2488-9849

Journal DOI: 10.46654/ij.24889849

Article DOI: 10.46654/ij.24889849.e6724

Z.H. AL-Tairi, R. M. (2014). Skin Detection Using YUV and RGB color spaces. Journal of Information Processing Sytems.

Zhongshui QU, J. W. (2010). A Color YUV Image Edge Detection Method Based on Histogram Equalization Transformation. Sixth inrernational Conference on Natural Computation. ICNC. 